\title{
Application and Reliability of Caprini Thrombus Risk Assessment Scale in Risk Assessment of Venous Thromboembolism in Acute and Severe Uygur Patients
}

\author{
Jinfang Yue', Lingyun Zuo², Xiang Sí2, Juan Chen² \\ ${ }^{1}$ Department of the First Intensive Care Unit, Critical Care Medicine, The First People's Hospital of Kashi District, Kashi, China \\ ${ }^{2}$ Department of Surgical Intensive Care Unit, The First Affiliated Hospital of Sun Yat-sen University, Guangzhou, China \\ Email: ayue97@hotmail.com
}

How to cite this paper: Yue, J.F., Zuo, L.Y., Si, X. and Chen, J. (2019) Application and Reliability of Caprini Thrombus Risk Assessment Scale in Risk Assessment of Venous Thromboembolism in Acute and Severe Uygur Patients. International Journal of Clinical Medicine, 10, 596-603. https://doi.org/10.4236/ijcm.2019.1011048

Received: October 7, 2019

Accepted: November 5, 2019

Published: November 8, 2019

Copyright $\odot 2019$ by author(s) and Scientific Research Publishing Inc. This work is licensed under the Creative Commons Attribution International License (CC BY 4.0).

http://creativecommons.org/licenses/by/4.0/

(c)

\begin{abstract}
Background: To explore the application and reliability of Caprini thromboembolism risk assessment scale in the risk assessment of venous thromboembolism in acute and severe uygur patients. Methods: 160 cases of acute and severe Uighur patients with venous thrombo embolism (VTE) that were treated in our hospital from December 2017 to December 2018 were selected as the research group. 160 cases of acute and severe uygur patients without VTE admitted to our hospital in the same period were selected as the control group. Caprini thrombus risk assessment scale and Padua thrombus risk assessment scale were used to evaluate in both groups. The general data of the two groups were compared. The results of the two groups were consistent using Caprini and Padua blood clot risk assessment scales. Clinical efficacy of two different thrombosis risk assessment scales in risk assessment of VTE. Results: Group and control group in the gender distribution, backlog of red blood cells and platelet count have no significant difference $(P>0.05)$, the team average age, average hospitalization days were significantly less than control group $(P<0.05)$, the team hemoglobin level was significantly higher than that of control group $(P<0.05)$, the team of white blood cells were significantly lower than control group $(P<0.05)$. Caprini thrombosis score was used to evaluate 156 high-risk patients and 164 low-risk patients. A total of 75 cases of high-risk patients and 245 cases of low-risk patients were assessed by Padua thrombosis score, and the results of the two assessment methods were significantly different $\left(\mathrm{X}^{2}=6.956, P<0.05\right)$. Consistency (Kappa coefficient) was 0.58 , indicating medium consistency. The specificity of Caprini thrombus score was significantly lower than that of Padua thrombus score $(P<0.05)$,
\end{abstract}


the sensitivity and negative predictive value of Caprini thrombus score were significantly higher than that of Padua thrombus score $(P<0.05)$, and there was no significant difference in the positive predictive value between the two groups $(P>0.05)$. Conclusion: The sensitivity, negative predictive value and positive predictive value of Caprini thrombosis risk assessment scale in VTE risk assessment of acute and severe uygur patients are very prominent, and the clinical efficacy is better, which is worthy of application.

\section{Keywords}

Venous Thromboembolism, Critically Ill Patients, Uygur, Risk Assessment, Clinical Effectiveness

\section{Introduction}

Venous Thrombus Embolism (VTE) is a common complication during the clinical treatment of critical ill patients [1]. Caprini thrombosis risk assessment scale was developed and named by Caprini, a foreign scholar. After it was introduced into domestic clinical practice, it was revised in 2009, and many large sample retrospective studies have been carried out in western clinical practice. Its feasibility and effectiveness have been proved [2]. However, most clinical studies have focused on medical or surgical patients, and studies using the Caprini thrombotic risk assessment scale for severely ill uyghur patients are very rare. This study applied the control-pathology method and retrospectively analyzed the VTE risk assessment of uyghur patients with acute and severe diseases by using Caprini thrombus risk assessment scale and Padua thrombus risk assessment scale, as reported below. The purpose of our study was to explore the application and reliability of Caprini thromboembolism risk assessment scale in the risk assessment of venous thromboembolism in acute and severe uygur patients.

\section{Data and Methods}

\subsection{General Information}

160 critical uygur patients with venous thrombo embolism (VTE) admitted to our hospital from December 2017 to December 2018 were selected as the study group. The mean age $(62.4 \pm 13.8)$ was 84 male patients and 76 female patients. Inclusion criteria: 1) Age $\geq 20$ years; 2) $>2 \mathrm{~d}$ was accepted in ICU; 3) Meet the clinical diagnostic criteria for acute and severe patients; 4) It meets the clinical diagnostic criteria for DVT; (5) complete previous clinical data.

160 critical uygur patients without VTE were selected as the control group. The mean age was $(51.6 \pm 11.9)$, including 87 years for males and 73 years for females. The study was approved by the ethics committee. Inclusion criteria: 1) Age $\geq 20$ years; 2 ) $>2 \mathrm{~d}$ was accepted in ICU; 3 ) No VTE occurred during hospitalization; 4) Complete previous clinical data. 


\subsection{Methods}

General data collection: gender, age, relevant laboratory biochemical indicators of the two groups were collected and analyzed retrospectively and recorded into the statistical system.

Padua thrombosis risk assessment scale: there are eleven risk factors in the scale.

The risk factors include: corticosteroid therapy, the obese (IBM 30 or higher), acute infections or rheumatoid disease, acute myocardial infarction, heart or lung failure, old age (more than 65 years), traumatic surgery within 30 days, high tendency to thrombosis disease, low mobility, history of VTE and active cancer patients. The score range of each of the 11 risk factors mentioned above was 1 point to 3 points, and the total score accumulation degree of all factors was divided into two grades: low risk: $\leq 3$ points, high risk: $>3$ points [3].

Caprini thrombus risk assessment scale: using the revised version of 2009, including 40 risk factors. Both control and study groups were assessed for thrombus risk. The assessment score range of each risk factor: 1 - 5 points. When there is no risk factor, 0 points will be scored. According to the assessment score, the risk rating and risk grading were carried out: low risk: $\leq 1$ point, medium risk: 2 points, high risk: 3 - 4 points, super high risk: $\geq 5$ points [4].

\subsection{Observation}

General data were observed and compared between the two groups. To observe and compare the consistency of assessment results between the two groups using Caprini thrombus risk assessment scale and Padua thrombus risk assessment scale. To observe and compare the clinical efficacy of two different thrombus risk assessment scales in risk assessment of VTE. Padua thrombus score included two grades: low risk: $\leq 3$, high risk: $>3$; Caprini thrombus score results include four levels: low risk: $\leq 1$ point, medium risk: 2 points, high risk: $3-4$ points, super high risk: $\geq 5$ points. When comparing the consistency of the above evaluation results, Caprini thrombus score was divided into two grades: low risk: $\leq 2$ points and high risk: $>2$ points, in order to facilitate the comparison of clinical efficacy of the two groups, including specificity, sensitivity, negative predictive value and positive predictive value. ROC analysis method was used for comparative calculation.

\subsection{Statistical Analysis}

Descriptive statistics were utilized to analyze for demographic, clinical, and outcome parameters. Continuous data are presented as the means and standard deviations (SDs) or medians and interquartile ranges. Categorical data are summarized as a total number and percentage of the cohort. All continuous variables were compared using Student's t-test or the Wilcoxon signed-rank test in the absence of a normal distribution. Categorical variables were compared using the Chi-squared test. A P-value $<0.05$ was considered statistically significant with 
95\% confidence intervals (CIs) also reported. These statistical analyses were performed using SPSS 19.0 software (SPSS, Chicago, IL, USA).

\section{Results}

\subsection{Characteristics}

There was no significant difference in the gender distribution, backlog of red blood cells and platelet count between study group and control group $(P>0.05)$. The average age, average hospitalization days in the study group was significantly less than control group $(P<0.05)$, the hemoglobin level in the study group was significantly higher than that of control group $(P<0.05)$, the white blood cells in the study group were significantly lower than control group $(P<$ 0.05). The characteristic was seen in Table 1.

\subsection{Consistency of the Two Groups}

Consistent results between the two groups were evaluated, using the Caprini thrombotic risk assessment scale and the Padua thrombotic risk assessment scale. Caprini thrombus score was used to evaluate 156 patients with high risk and 164 patients with low risk. A total of 75 patients with high risk and 245 patients with low risk were evaluated by Padua thrombus score. The results of the two assessment methods were significantly different $\left(\mathrm{X}^{2}=6.956, P<0.05\right)$. The Kappa coefficient was 0.58 , indicating moderate consistency. See Table 2 for details.

\subsection{Clinical Efficacy of Two Scales}

The clinical efficacy of two different scales was compared in risk assessment of VTE.

The specificity of Caprini thrombus score was significantly lower than Padua thrombus score $(P<0.05)$. The sensitivity and negative predictive value of Caprini thrombus score was significantly higher than Padua thrombus score $(P$ $<0.05)$, and there was no significant difference in the positive predictive value between the two groups $(P>0.05)$, the results were shown in Table 3 .

\section{Discussion}

Clinical VTE is divided into two types: Pulmonary Embolism (PE) and Deep Vein Thrombosis (DVT) [5]. Generally speaking, the condition of severe

Table 1. Characteristics of control and study groups.

\begin{tabular}{ccccccccccc}
\hline \multirow{2}{*}{ Group } & Case & \multicolumn{2}{c}{ Gender } & Age & hospital days & hemoglobin & WBC & HCT & PLT \\
\cline { 2 - 11 } & \multicolumn{3}{c}{ Male } & Femal & $($ year, $\bar{x} \pm s)$ & $(\mathrm{d}, \bar{x} \pm s)$ & $(\mathrm{g} / \mathrm{l}, \bar{x} \pm s)$ & $\left(\times 10^{9} / 1, \bar{x} \pm s\right)$ & $(\%, \bar{x} \pm s)$ & $\left(\times 10^{9} / l, \quad \bar{x} \pm s\right)$ \\
\hline Control & 160 & 87 & 73 & $51.6 \pm 11.9$ & $11.8 \pm 2.6$ & $131.6 \pm 16.5$ & $6.8 \pm 2.1$ & $38.4 \pm 3.7$ & $198.5 \pm 48.2$ \\
Study & 160 & 84 & 76 & $62.4 \pm 13.8$ & $17.6 \pm 2.1$ & $124.9 \pm 36.1$ & $10.7 \pm 4.8$ & $37.2 \pm 4.1$ & $197.6 \pm 47.6$ \\
$\chi^{2} / \mathrm{t}$ & $/$ & & 1.624 & 15.462 & 13.274 & 11.837 & 16.649 & 1.362 & 1.023 \\
$P$ & $/$ & & $>0.05$ & $<0.05$ & $<0.05$ & $<0.05$ & $<0.05$ & $>0.05$ & $>0.05$ \\
\hline
\end{tabular}


Table 2. Consistency evaluation between the two groups using Caprini scale and Padua scale.

\begin{tabular}{ccccc}
\hline & \multicolumn{4}{c}{ Caprini thrombus risk assessment scale } \\
\cline { 2 - 5 } & & High risk & Low risk & Total \\
\hline $\begin{array}{c}\text { Padua thrombus risk } \\
\text { assessment scale }\end{array}$ & High risk & 70 & 5 & 75 \\
& Low risk & 86 & 159 & 245 \\
& Total & 156 & 164 & 320 \\
\hline
\end{tabular}

Table 3. Clinical efficacy of two different thrombotic risk assessment scales in risk assessment of VTE.

\begin{tabular}{ccccc}
\hline Risk assessment scales & sensitivity & specificity & $\begin{array}{c}\text { negative } \\
\text { predictive value }\end{array}$ & $\begin{array}{c}\text { positive } \\
\text { predictive value }\end{array}$ \\
\hline Caprini scale & $73.4 \%$ & $70.9 \%$ & $71.2 \%$ & $72.3 \%$ \\
Padua scale & $85.6 \%$ & $32.2 \%$ & $55.8 \%$ & $69.1 \%$ \\
$\mathrm{X}^{2}$ & 6.294 & 7.023 & 5.493 & 0.516 \\
$\mathrm{P}$ & $<0.05$ & $<0.05$ & $<0.05$ & $>0.05$ \\
\hline
\end{tabular}

emergency patients is relatively serious and critical. During the course of clinical treatment, patients may suffer from coma, dehydration, infection and long-term postoperative limb immobilization [6]. Patients with severe condition may even have combined cardiopulmonary dysfunction, thereby increasing the risk of VTE [7]. The American College of Chest Physicians (ACCP), in the 9th edition of its clinical practice guidelines on antithrombotic and thromboembolic prophylaxis, proposed that all patients with acute and severe clinical conditions should be assessed for VTE risk during clinical treatment [8]. For patients with high risk of VTE, corresponding preventive intervention measures should be applied to reduce the rate of patients with VTE [9]. Therefore, it is of great clinical significance to select a reliable, comprehensive and rapid VTE risk assessment scale.

The results of this study suggested that the sensitiity of risk assessment for Padua thrombi scale was low with only $32.2 \%$, suggesting that its predictive effect was not very satisfactory when assessing the risk of VTE in critically ill patients. This is consistent with relevant other research results [10]. VTE risk grading based on the Padua risk assessment scale was less effective and reasonable.

In the 1990s, a foreign physician and scholar named Caprini proposed for the first time to assign values to patients with existing thrombotic risk factors, classify patients according to the score, and implement different preventive interventions for patients according to different grades [11]. In the clinical guidelines of ACCP, Caprini thrombus risk assessment scale was a prediction and screening tool for clinical surgical patients with the risk of VTE. Although there is no rigorous statistical calculation method, it can provide a more reasonable classification for clinical patients in the process of risk assessment of VTE, and it is very simple and easy to use [12]. 
The results of our study indicated that there was a significant difference between the two evaluation methods $\left(\mathrm{X}^{2}=6.956, P<0.05\right)$. The sensitivity and negative predictive value of Caprini thrombus score was significantly higher than Padua thrombus score $(P<0.05)$. Consistent with the results of similar studies, the validity and reliability of risk assessment using the Caprini thromboembolic risk assessment scale for patients with acute and critical illness is significantly higher. The reason may be that the content and quantity of relevant risk factors included in the two different assessment scales are inconsistent. The Caprini thrombotic assessment scale includes a total of 40 risk factors, while the Padua thrombotic assessment scale only includes 11 risk factors. As a result, many related VTE risk factors in the Padua thrombus risk assessment scale could not be effectively reflected and applied in the evaluation process of patients, thereby reducing the effective prediction rate of patients at high risk of thrombus [13]. A study reviewed the medical records of a certain VTE patient (65-year-old male and a BMI of $29 \mathrm{~kg} / \mathrm{m}^{2}$ ). Clinical diagnosis was acute pancreatitis and central venous catheterization was used. After the patient was assessed using Caprini thrombotic risk assessment scale, the score was 6, indicating the risk of VTE with high risk. However, the Padua thrombi risk assessment scale was used to reassess the condition, with a score of 1 , indicating a low risk of VTE [14].

The results of our study suggested that the study group and control group has no significant difference $(P>0.05)$ in the gender distribution, backlog of red blood cell and platelet count. The average age and hospitalization days in the study group was significantly less than control group $(P<0.05)$, the hemoglobin level in the study group is significantly higher than that in control group $(P<$ $0.05)$, the white blood cells in the study group were significantly lower than that in the control group $(P<0.05)$. The study results confirmed that the risk factors influencing the occurrence of VTE in patients with acute and severe clinical diseases included age, hemoglobin level, white blood cells, etc. This is consistent with relevant research results [15]. At the same time, the length of hospital stay in the study group was significantly longer than that in the control group, which indicated that the occurrence of VTE in the patients would have a serious adverse impact on the recovery speed and recovery quality of the patients. Therefore, VET risk assessment of patients is conducive to the early prevention and intervention of patients with high risk of VTE, which can effectively reduce the risk of VTE in patients. At the same time, VET risk assessment can also speed up the recovery of patients.

In conclusion, when applying Caprini thromboembolism risk assessment scale to evaluate the risk of VTE in critical uygur patients, the sensitivity, negative predictive value and positive predictive value of the assessment are very outstanding, and the clinical evaluation is more effective and worthy of application.

\section{Financial Support and Sponsorship}

This work was supported by the grants from Guangdong Science and Technolo- 
gy Planning Project (2017B 020247002)

\section{Conflicts of Interest}

There are no conflicts of interest.

\section{References}

[1] Zou, Y.L., Wu, J.B., Hu, J.F., et al. (2017) Validity of Caprini Risk Assessment Scale for Assessing Risk of Venous Thromboembolism in Pregnancy and the Puerperium. Chinese Journal of Practical Gynecology and Obstetrics, 4, 1178-1182.

[2] Lin, C.P. and Fu, W.G. (2017) The Risk Assessment Tools and Assessments Perioperative Venous Thromboembolism in a General Surgical Patient. Chinese Journal of Practical Surgery, 37, 108-109.

[3] Kellie, Y. and Robert, W. (2017) Evaluation and Management of Acute and Chronic Portal Vein Thrombosis in Patients with Cirrhosis. Clinical Liver Disease, 10, 152-156. https://doi.org/10.1002/cld.679

[4] Hu, Z.H., You, G.L., He, M., et al. (2018) Value of Capfini Risk Assessment Model in Screening for Venous Thromboembolism among Patients Lying in Bed in the Department of Neurosurgery. Journal of International Neurology and Neurosergery, 45, 11-14.

[5] Hsu, C., Brahmandam, A., Brownson, K., et al. (2018) The Effects of Statin Therapy on Thrombus Resolution in Patients with Deep Venous Thrombosis. Journal of Vascular Surgery Venous \& Lymphatic Disorders, 6, 291-292. https://doi.org/10.1016/j.jvsv.2017.12.034

[6] Liu, S.N., Lu, S.L., Gu, Z.Y., et al. (2017) Risk Factors Analysis of Venous Thromboembolism in Post-Operative Patients with Gynecological Malignant Tumor and Application of Related Risk Assessment Table. Academic Journal of Second Military Medical University, 38, 1244-1249.

[7] Chen, Y., Zhou, H.X., Hu, Y.H., et al. (2017) Risk Factors of Pulmonary Embolism in Senile and Non-Senile Inpatient and the Predictive Value of Caprini Risk Assessment Model in These Two Populations. National Medical Journal of China, 97, 1200 .

[8] Vazquez-Garza, E., Jerjes-Sanchez, C., Navarrete, A., et al. (2017) Venous Thromboembolism: Thrombosis, Inflammation, and Immunothrombosis for Clinicians. Journal of Thrombosis and Thrombolysis, 44, 377-385. https://doi.org/10.1007/s11239-017-1528-7

[9] Liu, F.L. (2017) Comparative Analysis of Risk Assessment of Venous Thromboembolism between American College of Chest Physicians Evidence-Based Clinical Practice Guidelines and British National Institute for Health and Care Excellence Guidelines. Chinese Journal of Practical Surgery, 37, 119-124.

[10] Song, C.F., Li, H., Tian, B., et al. (2018) Incidence of Postoperative Venous Thromboembolism after Thoracic Surgery and Its Characteristic: A Single Center, Prospective Cohort Study. Chinese Journal of Surgery, 56, 284-288.

[11] Song, J.H., He, X., Lou, W.S., et al. (2017) Application of Percutaneous AngioJetthrombectomy in Patients with Acute Symptomatic Portal and Superior Mesenteric Venous Thrombosis. National Medical Journal of China, 97, 991-995.

[12] Peng, K.W., Zhang, Q., Liu, J.Y., et al. (2017) Venous Thromboembolism Prevention for Peritoneal Carcinomatosis Patients Treated with Cytoreductive Surgery and Hyperthermicin Traperitoneal Chemotherapy. Chinese Journal of Clinical Oncolo- 
gy, 44, 39-44.

[13] Wei, X.X., Yang, L.M., Cai, J.Q., et al. (2017) Investigation and Analysis of Present Status for Preventing Venous Thromboembolism in Cancer in Patients. China Journal of Hospital Pharmacy, 37, 1185-1188.

[14] Gao, Q.Q., Lu, S.S., Xu, X.X., et al. (2017) Quantitative Assessment of Hyperacute Cerebral Infarction with Intravoxel Incoherent Motion MR Imaging: Initial Experience in a Canine Stroke Model. Journal of Magnetic Resonance Imaging, 46, 550-556. https://doi.org/10.1002/jmri.25556

[15] Wang, X.D., Huang, J., Zhao, B.B., et al. (2018) Comparison of the Predictive Value of Different Risk Assessment Model in Gynecology Oncology Patients with Deep Vein Thrombosis. Progress in Obstetrics and Gynecology, 27, 12-16. 